\section{Nonsense-mediated decay mechanism is a possible modifying factor of clinical outcome in nonsense cd39 beta thalassemia genotype}

\author{
Maria Concetta Renda, ${ }^{1}$ Angela Vitrano, ${ }^{1}$ \\ Massimo Attanasio, ${ }^{2}$ \\ Emanuela Fecarotta, ${ }^{1}$ Angela Piazza, ${ }^{1}$ \\ Antonino Giambona, ${ }^{1}$ \\ Germana Fiorentino, ${ }^{1}$ Disma Renda, ${ }^{1}$ \\ Paolo Rigano, ${ }^{1}$ Giuseppina Calvaruso, ${ }^{1}$ \\ Filippo Cassarà, ${ }^{1}$ Aurelio Maggio' \\ 1U.O.C. Ematologia per le Malattie Rare \\ del Sangue e degli Organi Ematopoietici, \\ Ospedali Riuniti Villa Sofia-Cervello, \\ Palermo; 'Dipartimento di Scienze \\ Statistiche e Matematiche, Università \\ degli Studi di Palermo, Italy
}

\section{Abstract}

Nonsense-mediated mRNA decay (NMD) is a surveillance system to prevent the synthesis of non-functional proteins. In $\beta$-thalassemia, NMD may have a role in clinical outcome. An example of premature translation stop codons appearing for the first time is the $\beta$-globin cd39 mutation; when homozygous, this results in a severe phenotype. The aim of this study was to determine whether the homozygous nonsense cd39 may have a milder phenotype in comparison with IVS1,nt110/cd39 genotype. Genotypes have been identified from a cohort of 568 patients affected by $\beta$-thalassemia. These genotypes were compared with those found in 577 affected fetuses detected among 2292 prenatal diagnoses. The nine most common genotypes, each with an incidence rate of $1.5 \%$ or over, and together accounting for $80 \%$ of genotype frequencies, underwent statistical analysis. Genotype prevalence was calculated within the overall group. Results are expressed as proportions with $95 \%$ confidence intervals; $P \leq 0.05$ was considered statistically significant. A binomial distribution was assumed for each group; z-tests were used to compare genotype frequencies observed in the patient group with frequencies in the affected fetus group. In the absence of selecting factors, prevalence of these two genotypes was compared between a cohort of $568 \beta$-thalassemia patients (PTS) and 577 affected fetuses (FOET) detected during the same period. IVS1,nt110/cd39 was significantly more prevalent in FOET than PTS $(\mathrm{P}<0.0001)$, while there was no significant difference in prevalence of cd39/cd39 in FOET compared with PTS $(\mathrm{P}=0.524)$. These results suggest a cd39 genotype NMD mechanism may be associated with improved clinical outcomes in thalassemia major.

\section{Introduction}

Messenger RNA (mRNA) stability is a critical determinant that affects gene expression. Studies in yeast and other model eukaryotic organisms have revealed many pathways to modulate mRNA stability in response to developmental, physiological and/or environmental stimuli. $^{1}$

Nonsense-mediated mRNA decay (NMD) is a post-transcriptional process in eukaryotic cells by which transcripts from premature translation stop codons (PTCs) ${ }^{2}$ are recognized by NMD factors and rapidly degraded. ${ }^{3}$

The exact molecular mechanism for NMD in higher eukaryotes is still being investigated. Degradation occurs when the nonsense codon is at least 50-55 nucleotides upstream of the exon-exon junction. ${ }^{4,5}$

Nonsense and frameshift mutations are present in approximately one-third of point mutations that cause human genetic disorders. ${ }^{6}$ Phenotypes of genetic diseases are, therefore, likely to be frequently affected by NMD. NMD can affect clinical outcome in at least three important ways: i) altering the pattern of inheritance; ii) causing distinct traits to manifest from mutations in the same gene; and iii) modifying specific clinical phenotypes. ${ }^{3}$

$\beta$-thalassemia is a genetic disease in which PTCs may have a role in specific homozygous genotype for the nonsense cd39 mutation, described as a severe phenotype.

Furthermore, $\beta$-thalassemia is the first genetic disorder in which it was shown how NMD is involved in the clinical outcome of genetic disorders: NMD-activating nonsense mutations result in a recessive mode of inheritance with asymptomatic heterozygous carriers, whereas nonsense mutations bypassing the NMD mechanism are characterized by a dominant mode of inheritance resulting in a rare form of symptomatic heterozygous $\beta$-thalassemia. ${ }^{7,8}$

However, whether the $\beta$-thalassemia NMD mechanism in homozygous nonsense cd39 may be involved in improving clinical outcomes is not yet known.

Therefore, the aim of this study was to determine whether the homozygous nonsense cd39 mutation, because of a PTC mechanism, may have a milder phenotype in comparison with that of a heterozygous cd39/IVS1,nt110.

Furthermore, the translation of aberrant IVS1,nt110 $\beta$-globin mRNA to detect the presence of NMD was analyzed.
Correspondence: Aurelio Maggio, U.O.C. Ematologia per le Malattie Rare del Sangue e degli Organi Ematopoietici, Ospedali Riuniti Villa Sofia-Cervello, via Trabucco 180, 90146 Palermo, Italy.

Tel. +39.91.688.5251 - Fax: +39.91 .688 .0228 .

E-mail: aurelio.maggio@ospedaliriuniti.it

Key words: nonsense-mediated mRNA decay, beta-thalassemia, clinical outcame, beta-globin gene mutations.

Acknowledgments: the authors would like to thank the Foundation Franco and Piera Cutino for its support.

Contributions: AM, MCR, research coordination and manuscript writing; $\mathrm{AV}, \mathrm{MA}$, statistical analysis; AP, EF, AG, GF, MCR, FC, laboratory investigation; DR, PR, GC, patients recruitment; AM, paper principal investigator and responsible.

Conflict of interests: the authors declare no potential conflict of interests.

Received for publication: 24 May 2012.

Revision received: 23 August 2012.

Accepted for publication: 28 September 2012

This work is licensed under a Creative Commons Attribution 3.0 License (by-nc 3.0).

(C) Copyright M.C. Renda et al., 2012

Licensee PAGEPress, Italy

Thalassemia Reports 2012; 2:e2

doi:10.4081/thal.2012.e2

\section{Materials and Methods}

In the absence of selecting factors, prevalence of homozygous nonsense cd39 and compound IVS1,nt110/cd39 genotypes, was compared between a cohort of 568 affected $\beta$-thalassemia patients (PTS) and 577 affected fetuses (FOET) detected during the same period among 2292 prenatal diagnoses.

In the absence of selection bias, the prevalence of $\beta$-thalassemia molecular defects in affected fetuses should be similar to that found in $\beta$-thalassemia patients.

Possible biases influencing these findings could include: i) the effects of improvements in transfusion and chelation therapy in the different genotypes; and ii) prenatal diagnosis sought by some at-risk couples with differentiated genotypes. It is evident that neither bias currently exists, reflecting that both management of thalassemia major and prenatal diagnosis programs are carried out independently of thalassemia genotypes.

Therefore, any significant difference in the prevalence of these genotypes between fetuses and adults would be caused by the effect of genotype on phenotype. 


\section{Genotype analysis}

Since 1993, genotypes have been identified at the Hematology Unit for Rare Blood and Hematopoietic Organ Diseases, at the Riuniti Villa Sofia-Cervello Hospital, Palermo, Italy, from a cohort of 568 patients affected by $\beta$ thalassemia syndromes. These genotypes were compared with those found in 577 affected fetuses detected during the same period among 2292 prenatal diagnoses performed by chorionic villi sampling and DNA molecular analysis. The diagnosis of thalassemia was based on accepted clinical and molecular criteria. ${ }^{9,10}$

\section{Statistical analysis}

Ninety-four different genotypes were found among the two groups. The nine most common genotypes, each with an incidence rate of $1.5 \%$ or over, and together accounting for $80 \%$ of detected genotype frequency, underwent statistical analysis (Table 1). Affected fetuses with one of these genotypes were labeled FOET (297 fetuses), and affected $\beta$ thalassemia patients were labeled PTS (313 patients) (Figure 1). Genotype prevalence was calculated within the overall group (Table 1). If genotype impairs phenotype, in the absence of selecting factors, its prevalence should be higher in FOET than in PTS. Instead, if genotype improves phenotype, its prevalence should be higher in PTS than in FOET.

The analysis is mainly based on a comparison between genotype cd39/IVS1,nt110 and genotype cd39/cd39. Both genotypes identify a cohort of patients with thalassemia major who, over the last few years, received the same chelation treatment and transfusions. No patients included in this analysis showed a transition from thalassemia major phenotype to thalassemia intermedia phenotype. The patients included in this cohort were negative for the deletions of alpha globin gene -3.7, -4.2 and -MED, and for the HPFH mutations of G-gamma globin gene promoters. The G-gamma -158 mutation was not present in the cohort of patients with thalassemia major, since this variant is in linkage with the mutation IVS1,nt6, whose presence resulted in a statistically significant difference between the two groups, FOET versus PTS (Table 1).

Results are expressed as proportions with 95\% confidence intervals (CI); $\mathrm{P} \leq 0.05$ was considered statistically significant. A binomial distribution was assumed for each group; ztests were used to compare genotype frequencies observed in the patient group with frequencies in the affected fetus group. ${ }^{11}$ Statistical tests were performed using STATA 9.2 software (StataCorp, College Station, TX, USA)

\section{CDNA analysis}

Total RNA was extracted from IVS1,nt110 homozygous $\beta$-thalassemia patients with Trizol reagent (Gibco-BRL, Gaithersburg, MD, USA). Reverse transcription was performed with $1 \mathrm{mg}$ mRNA, an oligo-dT primer, and 200U M-MLV. PCR of $\beta$-globin cDNA was performed using the following human beta-globin specific primers: forward-CTCCTGAGGAGAAGTCTGCCG and reverse-GCTCACTCAGTGTGGCAAAG. PCR products were investigated by direct genomic sequencing (CEQ8800Beckman).

\section{Results}

Table 1 shows genotype frequency in the PTS and FOET groups. There was no significant difference in genotype prevalence of homozygous nonsense cd39 genotype between FOET versus $\mathrm{PTS}(\mathrm{z}=-0.637, \mathrm{P}=0.524)$ (Table 1). Only IVS1,nt110/cd39 was significantly more prevalent in FOET than PTS $(\mathrm{z}=-4.344$, $\mathrm{P}<0.0001)$. Instead, the prevalence of IVS1nt6/IVS1nt6 genotype was significantly higher in PTS than in FOET (18\% vs $2 \%$; $\mathrm{z}=7.17, \mathrm{P}<0.00001$ ).

Figure 2 shows the sequencing of $\beta$-globin cDNA-PCR products from an IVS1,nt110 homozygous patient; interruption of the frame

Table 1. Comparison between prevalence of patient genotypes and genotypes of $\beta$-thalassemia affected fetuses (1993-2006).

\begin{tabular}{llcccc} 
Genotype & nPTS (\%) & nFOET (\%) & $Z$ & IC & $P$ \\
IVS1nt110-cod39 & $40(11.5)$ & $78(24)$ & -4.344 & $(-0.18 ;-0.07)$ & 0.00001 \\
IVS1nt110-IVS1nt1 & $14(4)$ & $22(6.8)$ & -1.606 & $(-0.06 ; 0.006)$ & 0.108 \\
\hline cod39-IVS2nt745 & $14(4)$ & $15(4.6)$ & -0.409 & $(-0.03 ; 0.02)$ & 0.682 \\
IVS1nt110-IVS1nt110 & $37(10.5)$ & $42(13)$ & -0.975 & $(-0.07 ; 0.02)$ & 0.330 \\
\hline cod39-IVS1nt1 & $10(2.9)$ & $16(4.9)$ & -1.397 & $(-0.05 ; 0.008)$ & 0.162 \\
cod39-cod39 & $45(12.9)$ & $47(14.5)$ & -0.637 & $(-0.06 ; 0.03)$ & 0.524 \\
\hline cod39-IVS1nt6 & $55(15.5)$ & $47(14.5)$ & 0.422 & $(-0.04 ; 0.06)$ & 0.673 \\
IVS1nt110-IVS1nt6 & $35(10)$ & $23(7.1)$ & 1.340 & $(-0.01 ; 0.07)$ & 0.180 \\
\hline IVS1nt6-IVSInt6 & $63(18)$ & $7(2)$ & 7.170 & $(0.11 ; 0.2)$ & $<0.0001$ \\
\hline
\end{tabular}

nPTS, number $\beta$-thalassemia patients; nFOET, number affected fetuses; z, z-test score; IC, immediate constituent analysis.

\section{Genotype of $568 \beta$-thalassemia patients and 577 affected $\beta$-thalassemia fetuses}

94 different genotypes were observed in the 2 groups

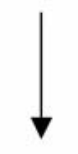

9 genotypes with incidence rate $\geq 1.5 \%$ were considered $(80 \%$ of detected genotypes frequency)

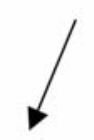

313 patients

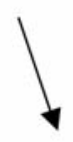

$297 \beta$-thalassemia affected fetuses

Figure 1. Profile of study to evaluate $\beta$-thalassemia genotypes found in the two groups considered. 


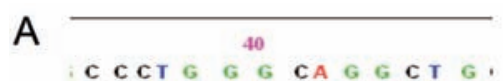

B
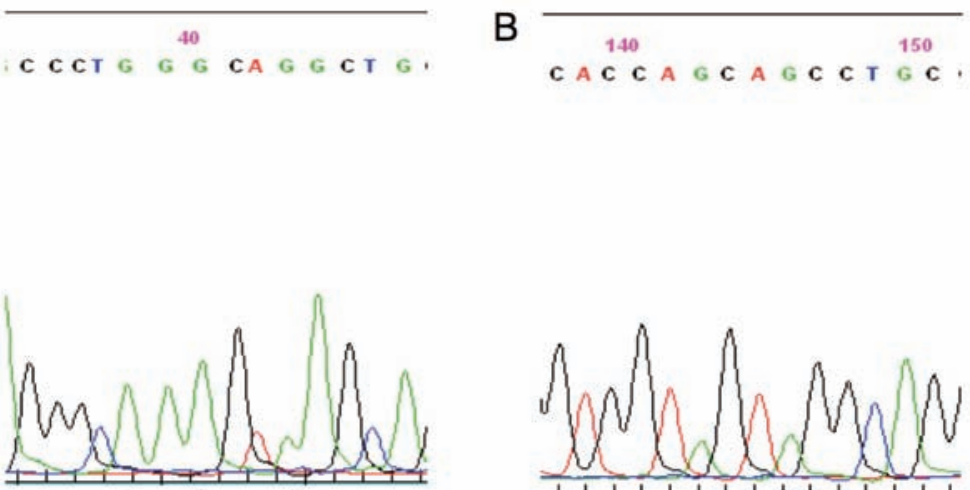

Forward strand
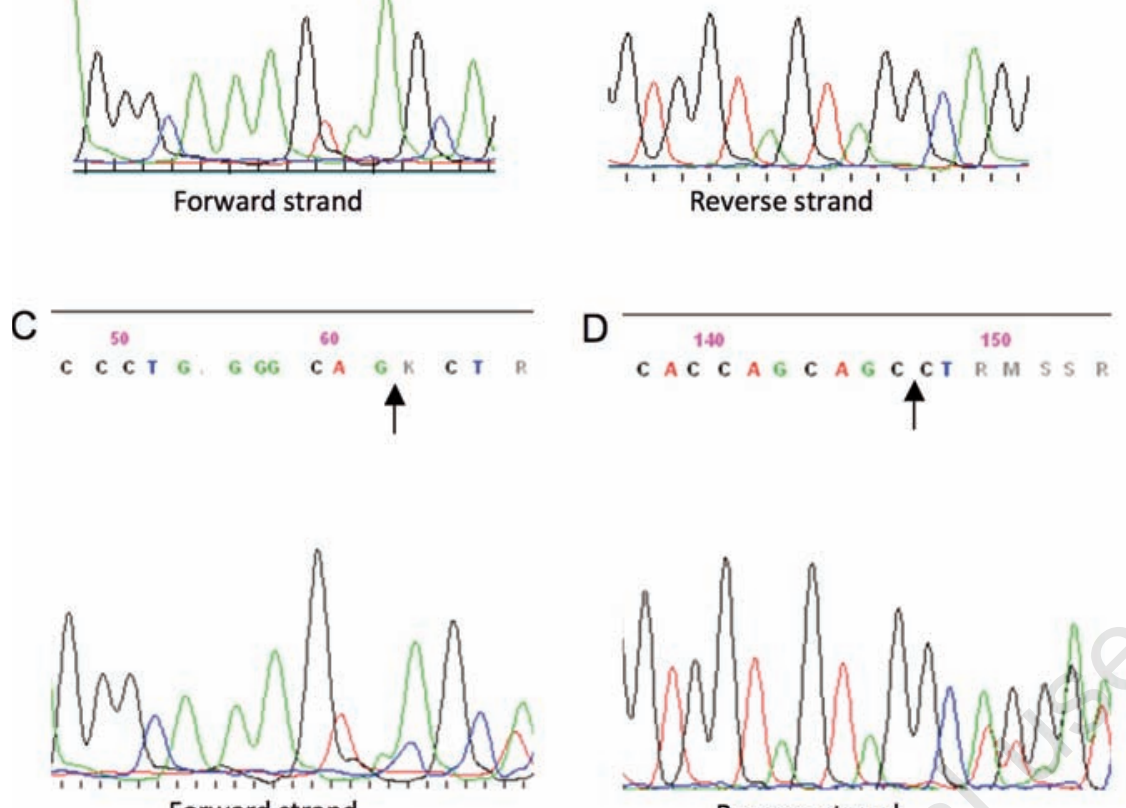

Forward strand

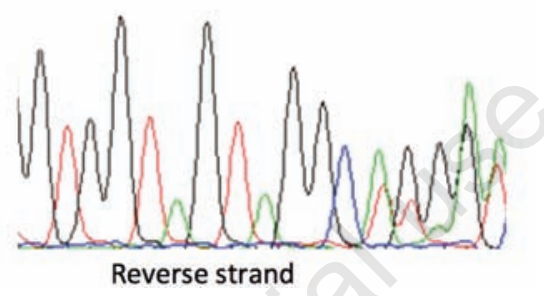

Reverse strand

more than 55 nt upstream of the next intronexon junction (Figure 1). ${ }^{12-15}$ The majority of abnormal $\beta$-globin transcripts in homozygous IVS1,nt110 could be due to their different clinical outcome in comparison with IVS1nt110/cd39 genotype (Table 1). Actually, in homozygous IVS1,nt110, the majority of abnormal $\beta$-globin transcripts could easily be metabolized or assembled into more functional protein complexes. The milder phenotype of IVS1,nt6, has been identified previously and was confirmed by these findings. ${ }^{16}$

The limitation of the study may be the presence of other genetic modifier factors influencing survival curves. ${ }^{17}$

In conclusion, the role of NMD as a modifier of the phenotypic consequences of PTC, already shown in other genetic diseases,${ }^{3}$ is becoming more apparent in $\beta$-thalassemia syndromes, and the position of a nonsense mutation alone is not sufficient to predict the fate of the affected mRNA and its effect on protein expression.

\section{References}

1. Isabel Peixeiro, Ana Luísa Silva, Luísa Romão. Control of human-globin mRNA stability and its impact on beta-thalassemia phenotype. Haematol 2011;96: 905-13.

Figure 2. Direct sequencing of $\beta$-globin cDNA-polymerase chain reaction products. (A and B) Normal subjects. (C and D) IVS1,nt110 homozygous patients. Interruption of the frame sequence (see arrows) indicates that both normal mRNA and abnormal mRNA containing a sequence 19 nucleotides longer, are present.

sequence indicates that both normal mRNA and abnormal mRNA containing a sequence 19 nucleotides longer are present.

\section{Conclusions}

Molecular defects of the $\beta$-globin gene have been well characterized and, to date, more than $200 \beta$-thal mutations have been described. ${ }^{12}$ It is well known that some $\beta$-thalassemia genotypes, such as nonsense cd39 homozygous, are especially severe, due to the complete lack of $\beta$-globin chain synthesis and the resulting imbalance of alpha-globin. Therefore, its prevalence in FOET would have been higher than in PTS (Table 1).

However, our findings suggest that the clinical outcomes of patients with cd 39 homozygous are no different from those in other severe genotypes with lower but present $\beta$-globin chain synthesis (Table 1), probably because of the NMD mechanism. ${ }^{3}$ NMD represents one of the major quality-control path- ways of the cell. In most described cases, NMD is splicing-dependent, always translationdependent, and relies on the interaction of the translation apparatus with the exon junction complexes or on the distance between the PTC. ${ }^{2}$ The more severe phenotype in the IVS1,nt110/cd39 genotype ( $\mathrm{z}=-4.344$, $\mathrm{P}<0.0001$ ) (Table 1) could be explained by the lack of NMD mechanism in the chromosome with the IVS1,nt110. This mutation produces abnormal $\beta$-globin transcripts, containing a sequence 19 nucleotides longer, inserting a new PTC (UAG) at nt16.

The molecular cDNA analysis of a small group of these patients confirms the lack of NMD mechanism in the chromosome with the IVS1,nt110 (Figure 1).

Our data show that, in $\beta$-globin IVS1,nt110 $\mathrm{G}>$ A mutation, NMD is probably disabled.

The expression of a human $\beta$-globin gene with the IVS1,nt110G>A mutation causes the presence of a faulty and frame-shifted mRNA, expected to encode anomalous and C-terminally truncated $\beta$-globin chains that are detrimental to cells, although the new insert PTC is
2. Danckwardt S, Neu-Yilik G, Thermann R, et al. Abnormally spliced b-globin mRNAs: a single point mutation generates transcripts sensitive and insensitive to nonsense-mediated mRNAdecay. Blood 2002; 99:1811-6.

3. Khajavi M, Inoue K, Lupski JR. Nonsensemediated mRNA decay modulates clinical outcome of genetic disease. Eur J Hum Genet 2006;14:1074-81.

4. Linde L, Boelz S, Neu-Yilik G, et al. The efficiency of nonsense-mediated mRNA decay is an inherent character and varies among different cells. Eur J Hum Genet 2007;15:1156-62.

5. Maquat LE. Nonsense-mediated mRNA decay in mammals. J Cell Sci 2005; 118:1773-6.

6. Mendell JT, Dietz HC. When the message goes away: disease-producing mutations that influence mRNA content and performance. Cell 2001;107:411-4.

7. Neu-Yilik G, Amthor B, Gehring NH, et al. Mechanism of escape from nonsensemediated mRNA decay of human b-globin transcripts with nonsense mutations in the first exon. RNA 2011;17:843-54.

8. Cao A, Galanello R, Rosatelli MC. Genotype-phenotype correlations in betathalassemias. Blood Rev 1994;8:1-12.

9. Modell B, Berdoukas V. The clinical 
approach to thalassemia. New York, NY: Grune and Stratton; 1984.

10. Maggio A, Giambona A, Cai SP, et al. Rapid and simultaneous typing of hemoglobin $\mathrm{S}$, hemoglobin $\mathrm{C}$ and seven Mediterranean beta-thalassemia mutations by covalent reverse dot-blot analysis: application to prenatal diagnosis in Sicily. Blood 1993;81:239-42.

11. Collett D. Modelling binary data. Boca Raton, FL: Chapman \& Hall/CRC Press; 1991.
12. Weatherall DJ, Clegg JB. The thalassemia syndromes. Oxford: Blackwell Science; 2001.

13. Maggio A, Caronia F, Orlandi F. Prenatal diagnosis of haemoglobinoathies in Sicily. Lancet 1992;339:1361-2.

14. Wittkopp N, Huntzinger E, Weiler C, et al. Nonsense-mediated mRNA decay effectors are essential for zebrafish embryonic development and survival. Mol Cell Biol 2009;29:3517-28.

15. Behm-Ansmant I, Kashima I, Rehwinkel J, et al. mRNA quality control: an ancient machinery recognizes and degrades mRNAs with nonsense codons. FEBS Lett 2007;581:2845-53.

16. Rosatelli MC, Oggiano L, Battista Leoni G, et al. Thalassemia intermedia resulting from a mild beta-thalassemia mutation. Blood 1989;73:601.

17. Danjou F, Anni F, Perseu L, et al. Genetic modifiers of $\beta$-thalassemia and clinical severity as assessed by age at first transfusion. Haematologica 2012;97:989-93. 